\title{
Philosophiques
}

\section{Entretien avec Monsieur Venant Cauchy}

\section{Émile Chrétien}

Volume 17, numéro 1, printemps 1990

URI : https://id.erudit.org/iderudit/027107ar

DOI : https://doi.org/10.7202/027107ar

Aller au sommaire du numéro

Éditeur(s)

Société de philosophie du Québec

ISSN

0316-2923 (imprimé)

1492-1391 (numérique)

Découvrir la revue

Citer ce document

Chrétien, É. (1990). Entretien avec Monsieur Venant Cauchy. Philosophiques, 17(1), 127-141. https://doi.org/10.7202/027107ar d'utilisation que vous pouvez consulter en ligne.

https://apropos.erudit.org/fr/usagers/politique-dutilisation/ 
PHILOSOPHIQUes, Vol. XVII, Numéro 1, Printemps 1990

\title{
INTERVENTION
}

\author{
ENTRETIEN AVEC \\ MONSIEUR VENANT CAUCHY
}

\section{PRÉSENTATION}

Le Congrès Mondial de Philosophie, tenu à Montréal en 1983 et présidé par $\mathrm{M}$. Venant Cauchy, a été important à bien des égards.

Il a permis, entre autres, de manifester à un moment propice l'ampleur de la réflexion philosophique, son sérieux et la place qu'elle occupe dans la culture.

C'est là, également, que plusieurs Québécois prirent connaissance de la méthode d'enseignement de la philosophie aux enfants de Matthew Lipman qui intéressera pendant plusieurs années la Société de Philosophie du Québec et fait aujourd'hui l'objet d'une expérimentation dans plusieurs écoles primaires de Montréal et d'ailleurs.

Il faut rappeler des "retombées" de cette nature pour mieux apprécier la tenue de tels évènements et rendre hommage à celles et à ceux qui y travaillent. Les nombreux contacts à l'étranger, l'implication dans des organismes internationaux de philosophie, constituent une ouverture sur le monde qui est bien réelle mais peut-être pas suffisamment connue.

C'est dans cette perspective qu'Émile Chrétien, qui s'intéresse spécialement à l'histoire de la philosophie au Québec, nous propose, sous forme d'entrevue, ce portrait de Venant Cauchy. Laval.

Émile Chrétien enseigne la philosophie au Collège Montmorency à 
Émile Chrétien : Monsieur Venant Cauchy, parlez-nous de votre carrière; comment en êtes-vous venu à enseigner la philosophie dans le Québec des années cinquante?

Venant Cauchy : J'enseigne la philosophie à Montréal depuis 1957. Avant de venir à l'Université de Montréal, j'ai enseigné dix ans aux États-Unis. Après mon doctorat, obtenu ici en 1947, j'ai été professeur pendant quatre ans à l'Université de St-Louis au Missouri et ensuite deux ans dans une université de San Antonio au Texas, de 1951 à 1953, et puis quatre ans à l'Université Fordham de New-York.

En 1957, s'ouvraient de nouveaux postes à l'Université de Montréal. On a commencé à engager des professeurs laïcs. Le Père Régis qui était doyen de la Faculté de philosophie m'a offert un poste que j'ai accepté avec enthousiasme, alors même que j'étais sur le point de m'installer définitivement aux États-Unis.

Je suis donc revenu. Ici, il y avait beaucoup de choses qui se passaient : on venait de fonder l'Association Canadienne de Pbilosophie, un processus de libéralisation s'amplifiait... J'ai collaboré à la consolidation de l'A.C.P. J'ai participé à l'organisation de différents congrès: $j$ 'ai bien connu un homme exceptionnel qui est mort beaucoup trop tôt, Jean Racette, qui a été professeur plus tard au Centre des Études Universitaires à Trois-Rivières, les Pères Jean Langlois et Jacques Croteau qui présidèrent l'Association Canadienne de Philosophie pendant les années 60. En 1961, toujours à l'intérieur de l'Association Canadienne de Philosophie, j'ai proposé la fondation de la revue Dialogue que j'ai par la suite co-dirigée jusqu'en 1974. Entre temps tout en dirigeant Dialogue, j'étais devenu secrétaire de la Faculté de Philosophie, Directeur de l'enseignement de la philosophie au baccalauréat intra muros de l'Université de Montréal ; c'était quelque chose de très considérable, puisqu'il y avait là quelques milliers d'étudiants. Et puis après la fondation de département de philosophie, j'en suis devenu le deuxième directeur de 1970 à 1974. C'est dans le cadre de ce poste que j'ai organisé le grand Congrès de l'Association des Sociétés de Philosophie de Langue Française sur la Communication, en 1971, congrès fort animé auquel participèrent de 350 à 400 philosophes des pays francophones du monde et dont les Actes ont été publiés en deux volumes. 


\section{É.C.: Dans ces années-là la question nationale était d'actualité} au Québec...

V.C.: Je présidais la Société de Philosophie de Montréal qui organisait chaque année une grande journée de philosophie, en divers endroits, notamment au restaurant Hélène de Champlain sur l'Île Ste-Hélène. Nous tenions des colloques sur des problèmes d'actualité comme la réforme proposée par le rapport Parent, le problème de la pauvreté, etc. Il y eut justement une de ces rencontres sur le thème de l'indépendance du Québec. C'étaient des événements très importants et très animés et c'est à partir de ces activités de la Société de Philosophie de Montréal que nous eûmes l'idée de constituer une société pour l'ensemble du Québec. À l'occasion de la journée sur l'indépendance du Québec à laquelle participèrent Jacques-Yvan Morin, Alan Montefiore, Yvon Blanchard, journée réunissant une centaine de philosophes du Québec, nous avons fondé la Société de Philosophie du Québec. La grande majorité y était tout à fait favorable. Pendant ma dernière année à la direction du département, nous avons mis au point les statuts de la Société de Pbilosophie du Québec. Cette année-là, en 1973-1974, nous avions constitué un comité provisoire représentatif des collèges et des universités du Canada français. À la fin de l'année, en mai 1974, à l'A.C.F.A.S.., nous avons établi officiellement la Société de Philosophie du Québec.

Peu après, je partais en année sabbatique à Toulouse, en France. Ensuite, j'ai continué à œuvrer dans l'Association Canadienne de Philosophie et j'ai lancé l'idée d'inviter la Fédération Intemationale des Sociétés de Philosophie à tenir son prochain congrès mondial à Montréal en 1983. Au congrès de Dusseldorf, l'invitation avait été faite mais l'Assemblée générale avait préféré la Grèce, terre d'origine de la philosophie occidentale. Mais les Grecs, ayant eu des problèmes d'espace et de financement, se sont retirés au bout d'un an et la FISP nous a demandé si notre invitation tenait toujours. Nous avons accepté et grâce à l'Université de Montréal, nous avons organisé le XVII Congrès mondial qui réunit au Palais des Congrès de Montréal, sur le thème de Philosophie et Culture quelque 2500 participants de 75 pays. Ceci nous ouvrait plusieurs portes car la Fédération Internationale se trouve dans tous les pays du monde. Elle compte plus de cent sociétés membres, 
nationales et internationales. Après le Congrès mondial, l'organisateur principal devenait selon la tradition, président de la Fédération Internationale. J'en ai donc été président pendant cinq ans, ce qui m'a permis d'avoir des relations philosophiques fréquentes avec les différentes sociétés du monde, notamment avec les pays de l'Est, la Bulgarie, l'URSS, la Pologne, la Chine et les pays de l'Amérique latine.

\section{É.C.: Dans ces pays, les philosophes jouent-ils un rôle} important?

V.C.: Ils peuvent jouer un rôle très important. Remarquez qu'en Union Soviétique, où la Société de Philosophie de l'URSS organise le prochain congrès mondial en 1993, le président de cette société, Monsieur Yvan Frolov, est le principal conseiller de $M$. Gorbatchev et vient d'être nommé directeur du journal la PRAVDA. On voit tout de suite le rôle déterminant que peuvent jouer les philosophes en URSS.

Je citerai encore l'exemple de ce qui s'est produit en Pologne et en Bulgarie. La présence philosophique dans les mouvements de réforme est extrêmement forte et les liens qu'ils ont pu nouer avec l'étranger ont été déterminants à mon sens. Nous avons des contacts avec la Bulgarie depuis 1983. Nous organisons chaque année, tantôt au Québec, tantôt en Bulgarie des colloques bilatéraux Canada-Bulgarie, dont les Actes sont publiés, sur des problèmes philosophiques actuels, tels que la perception de l'altérité, les conceptions de la paix en philosophie, la diversité culturelle, le pluralisme philosophique. Nous avons reçu ici à l'Université de Montréal, l'été passé, un jeune philosophe bulgare qui est un des instigateurs du principal mouvement d'opposition dans son pays, Ecoglasnost.

É. C. : Les philosophes québécois ont-ils joué un rôle important dans l'bistoire culturelle?

V.C.: Je pense qu'effectivement nous avons joué un rôle très important. Habituellement, on pense que le Québec a commencé à se développer avec la réforme de l'enseignement issue du Rapport Parent, mais il faut remonter beaucoup plus loin pour apprécier les influences, les germes de changement qui se manifestaient dans plusieurs établissements collégiaux de même qu’à 
l'Université de Montréal et à l'Université Laval. Malheureusement l'immaturité, l'inexpérience d'un certain nombre d'instigateurs de la réforme de l'enseignement, parfois même leurs préjugés idéologiques ont eu pour effet d'amener le Québec à supprimer ou à occulter un grand nombre d'acquis et de valeurs qui, avec des moyens très faibles, réussissaient à s'affirmer et qu'on aurait eu avantage à intégrer aux nouvelles institutions. On a agi comme si effectivement il n'y avait rien dans notre histoire qui aurait permis un effloraison, comme s' il fallait nier le passé. Je pense aux travaux des Dominicains comme les Pères Régis, Lachance, Forest, des hommes d'une grande ouverture d'esprit et à certains de nos collèges qui faisaient l'envie du Canada anglais. Je pense aussi à un Jésuite, le Père Racette, dont le dynamisme s'enracinait dans une lucide appréciation des traditions culturelles du Québec, mais dont le décès prématuré nous a privé d'un maître estimé dans la mise en œuvre des réformes.

Il y a eu sûrement une influence des philosophes québécois sur la prise de conscience des droits et de la dignité de la personne. Notre milieu n'était pas structurellement très fort; il a toujours été jusqu'à un certain point marginal. Mais un milieu marginal est un milieu beaucoup plus mobile, plus imprévisible. Il peut réagir plus rapidement et de manière plus décisive contre les forces de domination, de colonisation. C'est un milieu qui était quand même disposé à aller très loin. Effectivement le milieu a commencé à se prendre en main, à s'orienter d'une façon importante, à se penser et à refuser sa marginalisation. On a même qualifié ces changements de Révolution tranquille. Mais si tout cela a pu se produire, c'est sans doute en bonne partie sur la base d'une situation antérieure. Beaucoup de nos contemporains croient que le Québec actuel est sorti avec eux de la cuisse de Jupirer, qu'un grand déluge supprima le passé auquel l'état présent succéda comme par génération spontanée. Je pense qu'effectivement, il y avait eu beaucoup d'éléments novateurs, dont on ne tient pas assez compte, dans le passé du Québec. En effet, si l'on remonte avant la conquête, on retrouve une sorte d'affirmation des Canadiens vis-à-vis des Français de France, les Canadiens se considéraient déjà comme Canadiens, et non comme des Français, ce qui causait des frictions avec les fonctionnaires et administrateurs venus de France. Cela ne fut pas étranger à la défaite et à la chute de la Nouvelle-France en 17591760. Cette conscience nationale s'affirma également dans les 
rébellions de 1837-1838, durement réprimées par la puissance anglaise. Par la suite, il y eut beaucoup d'autres essais d'affirmation qui eurent pour conséquence paradoxale le développement d'un certain conservatisme philosophique et religieux. Les philosophes, comme philosophes professionnels, n'avaient pas de chance de s'exprimer sauf dans un cadre largement ecclésiastique. Ce fut, à mon avis, une des causes du conservatisme. La situation a commencé à s'ouvrir au XIXe siècle, puis dans les années 30 et 40 .

É.C. : Que pensez-vous de notre production philosophique actuelle?

V.C.: Elle me semble assez active. Elle n'est peut-être pas aussi développée, aussi reconnue qu'elle pourrait et devrait l'être. Si dans un milieu donné on ne reconnaît pas ce qui s'y fait, si l'on ne s'y intéresse pas, il y a peu de chance que cette reconnaissance vienne d'ailleurs. Une production locale qui n'est pas appréciée ou discutée dans son milieu d'origine, arrive difficilement à se développer et à s'affirmer. Les occasions ne manquent pas cependant, les possibilités d'action y sont, l'espace est là à occuper autant au plan international qu'au plan national. S'il n'est pas occupé, c'est que l'on n'est pas conscient de cet espace ou que l'on se replie sur soi. Par exemple, si la Société de Philosophie de Montréal n'avait pas lancé son invitation en 1968, il n'y aurait pas eu de congrès international à Montréal en 1971, sur la Communication. Il en est de même pour le congrès mondial de 1983 dont j'ai déjà parlé. Il faut savoir exploiter les occasions qui s'offrent.

\section{E.C.: Quel peut être le rôle des philosophes à notre époque?}

V.C.: Le rôle que je vois pour le philosophe québécois c'est de parler, d'écrire, d'intervenir là où ils le peuvent et le doivent. Il y a combien de problèmes, combien de questions qui leur sont posées ou qu'ils se posent, questions sur l'environnement, la guerre nucléaire, les développements de la génétique, avec leur potentiel de déshumanisation autant que de bienfaisance humaine, les disparités économiques Nord-Sud et Est-Ouest, les relations d'échanges internationaux fructueux à établir avec les pays de l'Est, etc. Ce que nous avons pu faire n'est qu'une fraction de ce qui pourrait se faire. Par exemple, tenter d'influencer les organismes gouvernementaux pour s'assurer qu'il y ait des services adéquats 
dans tous les domaines, la justice, l'éducation, les affaires sociales, tenter de faire prendre conscience aux gouvernements et aux groupes organisés des obligations et responsabilités d'une société à l'endroit de ses membres... Par exemple, j'ai pu accomplir un certain nombre de choses pendant mon mandat de cinq ans (1983-1988) à la tête de la Fédération Internationale des Sociétés de Philosophie (FISP). J'ai pu stimuler, accentuer la communication entre les philosophes des pays socialistes et de l'Occident. Nous avons intensifié l'échange d'information en utilisant les bons offices de publications chinoises, polonaises, latino-américaines, africaines, entre autres. J'ai encouragé dans divers congrès la prise de parole par des penseurs d'Afrique et d'Amérique latine, notamment ceux qui se rattachent à la philosophie de la libération. Nous avons institué des colloques annuels bilatéraux Bulgarie-Canada, dont les Actes ont été publiés. Au XVIII Congrès mondial de philosophie à Brighton au Royaume-Uni, j'ai proposé la fondation au sein de la FISP d'une Commission permanente de recherche interculturelle en philosophie dont l'un des objectifs consiste à réduire l'influence débilitante des ethnocentrismes (plus particulièrement l'eurocentrisme) sur la pensée philosophique, compte tenu des graves problèmes que posent à la survie de l'humanité l'esprit de guerre, la puissance croissante des technologies, les pollutions de toutes sortes issues en grande partie de la modernité occidentale... Cette Commission, que je préside, compte comme membres les professeurs Imamichi du Japon, Miro-Quesada du Pérou, Francis Jacques de France, Haddad-Chamak de Tunisie, Xing Fensi de Chine, N'Tumba du Zaïre. La Commission publie un bulletin annuel de ses activités et coordonne un certain nombre de groupes de recherche à Séville et Barcelone en Espagne, à Toulouse en France et ici à Montréal où le groupe de recherche que j'anime s'est proposé comme thème : la perception de l'altérité et la diversité culturelle.

Ces préoccupations se prolongent quant à moi dans ma participation à deux organismes, le Groupe de Vézelay sur les risques technologiques majeurs, qui songe à convoquer une convention au Québec en juillet 1992, et l'Association internationale d'échanges scientifiques sur la violence et la coexistence bumaine (ASEVICO) dont je préside le Comité exécutif et qui m'a confié l'organisation du $I^{e}$ Congrès Mondial sur la Violence et la Coexistence bumaine, qui aura lieu au Palais des Congrès de Montréal en 
1992. L'ASEVICO est une association multidisciplinaire à laquelle sont appelés à participer les spécialistes et chercheurs préoccupés par les problèmes de la vie sociale et la multiplicité des formes de violence.

É.C.: Que pensez-vous du rôle de professeur de philosophie?

V.C.: Qu'est-ce qu'être professeur de philosophie? C'est engager une réflexion fondamentale sur l'être humain, sur l'individu dans le monde, sur la société etc. Mais comment en rester là alors que la façon dont on existe, le bien-être, la qualité de la vie, la nature de la société dans laquelle on vit se trouvent déterminés par le contexte social et par les gouvernements. Les gouvernements sont d'ailleurs, dans une certaine mesure, inconscients de tout ça et s'il n'y a pas intervention des philosophes et du public en général, ils le demeurent. Il n'y a pas de scission entre la salle de cours et l'expression publique ou l'influence que l'on peut chercher à exercer. Il faut intervenir avec les moyens dont on dispose, et ces moyens sont beaucoup plus puissants qu'on pense. Comme je le disais, l'espace est là à occuper et, s'il n'est pas occupé par les gens qui peuvent penser ces problèmes en profondeur, il risque de l'être par des gens moins scrupuleux, par ceux que Socrate qualifiait jadis de sophistes...

E.C.: Oui, mais les philosophes ne sont pas les seuls à penser. Pouvez-vous spécifier la façon dont vous envisagez leur rôle?

V.C.: Par exemple, ce n'est pas le professeur de chimie comme tel, (même si ce qu'il fait est très intéressant, car il peut contribuer à la mise sur pied d'une usine, au bien-être économique du milieu et même à la conception de normes sur les substances polluantes dans une perspective éthique) qui est le mieux placé pour poser les questions les plus cruciales sur la conduite de la vie. C'est le philosophe qui, de par la nature de sa discipline, est le plus apter à poser des questions de fond. Vous savez, aujourd'hui, quand on parle de discipline de pointe, on pense à la haute technologie, ou à l'informatique, mais à mon sens, il y a une véritable discipline de pointe qu'on ignore, c'est la philosophie. C'est en philosophie que les problèmes les plus cruciaux, les problèmes fondamentalement humains se posent. La technologie 
ou la science ne sont pas à écarter, mais les sciences sans réflexion éthique et philosophique ont acculé l'humanité tout entière aux plus graves dangers et même à l'absurdité de l'autodestruction.

E. C.: Les philosophes ont beaucoup de pain sur la planche!

V.C.: Disons qu'ils ont à réfléchir en profondeur sur les questions les plus importantes qui se posent à l'être humain. Ils ont à s'impliquer parce que jusqu'à maintenant on vivait dans un monde d'abondance, en air, en ozone, etc. Que pouvions-nous faire? Nous étions comme des poussières réparties à la surface du globe, dans l'immensité du cosmos. Mais maintenant, on exploite les ressources de la terre au maximum, on la pollue, on invente des armes qui menacent l'équilibre même de la planète et peuvent avoir comme conséquence l'élimination pure et simple de la vie humaine. Anciennement les techniques dont on disposait étaient inoffensives d'un point de vue cosmique. C'était malgré tout quelque chose qui exprimait notre humanité ou notre inhumanité fondamentale. Par exemple, les guerres, les expéditions de colonisation entraînaient la mort, la torture, le vol, le viol etc. Des civilisations disparaissaient comme cela est arrivé en 1492 lorsque Colomb a «découvert » les Indes occidentales : on y a massacré des millions d'autochtones. Évidemment c'étaient des crimes horribles à notre échelle, mais cela n'avait pas encore de répercussions cosmiques. On est aujourd'hui arrivé à un point tel que si l'Union Soviétique et les États-Unis s'affrontaient, ou même si la France avec son petit arsenal s'avisait de lancer ses ogives nucléaires, on pourrait se trouver dans une situation apocalyptique à laquelle le monde humain ne pourrait survivre. On se rappelle Tchernobyl, un accident très ponctuel et très limité, dont les effets ont été ressentis non seulement en Bulgarie, en Pologne et à Moscou, mais même jusque dans le sud de l'Italie. losophes.

É.C. : De là l'urgence d'une prise de parole de la part des phi-

V.C.: Oui évidemment, c'est-à-dire une prise de parole comme philosophes. Pas à la façon du philosophe roi de Platon qui considère qu'il possède la vérité par le fait qu'il est philosophe, mais au contraire en réfléchissant profondément sur les problèmes humains d'une façon systématique, dans un contexte historique et selon des méthodes qui sont propres à notre discipline. C'est 
seulement la philosophie qui de par sa nature peut aborder ces questions avec toute la profondeur nécessaire. Disons qu'un chimiste, un physicien, un mathématicien peuvent s'intéresser de manière pertinente à des questions fondamentales, mais il n'en demeure pas moins que le philosophe a un rôle essentiel à jouer. J'entrevois cependant le danger d'une dérive de la philosophie dans le sens d'un désengagement, d'une sorte de scepticisme stérile qui la détourne de réfléchir aux véritables problèmes. Et dans la mesure où la philosophie canadienne ou québécoise, comme la philosophie nord-américaine va dans le sens d'un tel désengagement, où elle répugne à s'occuper de situations concrètes, où elle réduit son rôle à clarifier le sens des mots et des idées qu'on emploie, dans la mesure où elle s'en tient à des exercices purement formels, désincarnés, qu'elle refuse de se prononcer sur les problèmes individuels er sociaux, elle se condamne elle-même à l'impuissance et à l'insignifiance. Dans les pays qui sont les plus touchés par les problèmes sociaux (et ce ne sont pas seulement les pays de l'Est, l'Afrique ou l'Amérique latine), cette forme de dilettantisme formalisant et logicisant, réduit la philosophie à l'inexistence effective. C'est tout comme si l'on déclarait implicitement que les problèmes humains, la violence internationale, nationale ou individuelle, les dysfonctionnements sociaux, économiques et politiques, représentaient une sorte de fatalité que l'homme doit subir. $\mathrm{Ce}$ type de philosophie fait abstraction de la possibilité pour l'homme de réfléchir à sa condition et de s'en sortir par sa raison et son dynamisme.

En d'autres termes, ce n'est pas la philosophie comme telle qui est à délaisser, mais à l'intérieur de la philosophie elle-même, on doit en contester les déviations sophistiques comme le font par exemple certains philosophes latino-américains. Si en fait la raison humaine n'est pas capable de faire face aux problèmes qui nous hantent, qui nous écrasent effectivement, il n'y a rien à faire, on est à la dérive et ça va continuer comme ça. Une telle optique philosophique devient alors pour ceux qui souffrent gravement de ces problèmes, quelque chose d'insultant, une façon de dire que la philosophie n'est rien, ou n'est qu'une activité purement formelle qui doit s'abstenir de toute velléité de prise de parole ou d'intervention dans les problèmes qui les écrasent. À ce moment-là, on laisse la parole aux petits dictateurs, aux déviants qui occupent le 
haut du pavé, entre autres en Amérique latine et en Afrique. Un tel abstentionnisme apporte de facto et implicitement son appui à un statu quo intolérable. On peut citer à titre d'exemple de ce débat la prise de position du philosophe mexicain Léopoldo Zéa qui juge, d'une façon critique, le rôle d'une certaine philosophie nordaméricaine du point de vue d'un philosophe du Tiers-Monde, dans une section du périodique Cuardernos Americanos intitulé Imperialismo cultural (il m'a fait l'honneur d'y inclure en traduction espagnole, une conférence que j'ai prononcée au Congrès interaméricain de philosophie de Guadalajara, intitulée : "Consideraciones sobre la Ubicación y Viabilidad de Una Filosofia Real». La philosophie n'est pas une voie d'évitement, elle est au centre ou elle n'est pas. Elle est dans la problématique sociale, dans la problématique politique et économique, ou elle n'est nulle part.

É.C.: Quel rôle voyez-vous pour le professeur de philosophie, de C.E.G.E.P. en particulier?

V.C.: Le professeur de C.E.G.E.P. dans la mesure où son enseignement est obligatoire (ce qui n'est pas le cas à l'Université) est en mesure d'inculquer aux étudiants le sens de l'importance des questions éthiques et philosophiques afin qu'ils ne se satisfassent pas de ces illusions formalistes et formalisantes qui sont une perpétuelle tentation en philosophie.

Le philosophe a un rôle social à jouer, il doit poser sa pierre à l'édifice commun et, à mon sens, sa pierre est une pierre indispensable ; s'il ne la pose pas, c'est comme si la pierre d'angle manquait. On ne peut pas demander au philosophe de résoudre tous les problèmes de l'humanité mais il doit s'en préoccuper et s'efforcer d'indiquer des directions... Et il n'est sûrement pas en tant que philosophe plus limité que d'autres... D'ailleurs avec tout le respect et toute l'admiration que j'ai pour les mathématiciens, pour les spécialistes de sciences politiques, pour les sociologues, pour les chimistes, pour les spécialistes d'autres disciplines, je pense qu'euxmêmes reconnaissent le rôle spécifique du philosophe dans leur propre discours. En effet, lorsqu'ils achoppent sur une question fondamentale, sur laquelle leur méthode n'a pas de prise, ils renvoient à la philosophie, ou ils se font eux-mêmes philosophes. Par exemple, les mathématiciens, lorsqu'on les interroge au sujet de la quantité, de l'infini, de l'infiniment petit, etc. refusent de 
répondre, ou bien s'ils essaient d'y répondre, ils le font en tant que philosophes. Il en est ainsi, quel que soit le domaine. Ce fait ne devrait pas nous porter à devenir suffisants, car si l'on considère la philosophie comme une discipline portant sur les véritables questions fondamentales, nous restons avec nos limites, et nous devons en être conscients. Cependant il n'en demeure pas moins que comme philosophes, nous avons l'obligation de faire ce pour quoi la philosophie existe. Comme professeurs, c'est d'abord dans la salle de cours que nous pouvons agir, mais la salle de cours n'est pas tout, c'est seulement une ouverture sur un monde beaucoup plus large où nous ne pouvons pas ne pas nous impliquer.

\section{É.C. : Comme philosophe, on est impliqué dans la cité?}

V.C. : La cité comme telle se situe dans un ensemble et par la force des choses lorsqu'on parle de la cité, on parle aussi de ce qui lui est directement relié. On ne peut pas résoudre ses problèmes à soi comme individu seulement, parce que, effectivement, on est un individu dans l'ensemble social. On ne peut pas non plus aborder les problèmes sociaux en un sens étroitement national parce que la société nationale fait partie d'un ensemble. On ne peut même pas aborder les problèmes d'un point de vue purement conjoncturel ou intemporel parce que, effectivement, on se situe par rapport à un passé et en fonction d'un avenir. Qu'on le veuille ou non, les contextes plus larges nous englobent et nous situent, spatialement, temporellement et exitentiellement... Lorsque nous avons fondé la Société de Pbilosophie du Québec en 1974, notre but était justement de favoriser les échanges à la grandeur du Québec entre philosophes sur ces questions fondamentales. A cette époque, on nous objectait qu'en constituant une Société de Philosophie du Québec, nous nous séparions de l'Association Canadienne de Pbilosopbie, que nous versions dans une sorte de "séparatisme". Mais pour nous au contraire, exister comme ensemble cohérent, comme lieu d'échange, lieu de production, lieu d'activité, c'était renforcer un ensemble plus grand et de plus renforcer un ensemble international. Et en tant que fondateur de la S.P.Q., personne ne pouvait me faire ce reproche, puisque personne n'avait fait davantage pour consolider I'A.C.P. et pour intéresser notre milieu aux structures mondiales de la philosophie. 
É.C.: Est-ce que vous trouvez que les philosophes québécois sont à la bauteur?

V.C. : Ils ne peuvent se permettre de ne pas être à la hauteur. Cela dépend de la façon dont on se perçoit. C'est très important de se percevoir d'une certaine façon, comme capable d'intervenir. Ce qui m’a le plus frappé, moi qui avais fait toutes mes études ici, y compris mon doctorat et qui suis d'abord allé enseigner la philosophie aux États-Unis, c'est que je n'ai jamais eu l'impression que la qualité de ma formation philosophique ait été de quelque façon inférieure à ce que l'on faisait là-bas ou ailleurs dans le monde. Et il en fut de même lorsque j'ai enseigné à New York, ou lorsque j'ai participé activement aux travaux de grandes sociétés américaines de philosophie. Nous trouvions par exemple tout à fait normal que notre confrère, Hugues Leblanc, qui avait fait une licence en philosophie à l'Université de Montréal, puisse passer d'emblée à Harvard et y faire, en deux ans, je crois, sous la direction de Quine, un doctorat en logique moderne. Vous savez, si on ne sort pas de son milieu, on est peut-être porté à penser que ce milieu est inférieur à d'autres milieux. Ce qui est faux. Il faut savoir bien se percevoir pour entrer en rapport avec d'autres sur une base d'échanges fructueux et productifs au plan philosophique. Il ne faut pas se déconsidérer par rapport aux autres. J'ai toujours combattu vigoureusement tout au long de ma carrière le colonialisme culturel qui tend à déprécier son milieu et les siens. Il faut savoir apprécier ce que l'étranger peut nous apporter mais sans déconsidérer, comme on le fait trop souvent, ce que produit notre milieu culturel.

É.C.: De quelle façon percevez-vous l'avenir de la philosophie au niveau universitaire et dans le milieu québécois en général?

V.C. : À mon sens, l'avenir de la philosophie à l'université au Québec ou dans le milieu québécois, c'est en fait l'avenir de l'université elle-même. Si l'université s'atomise dans un ensemble de disciplines qui n'arrivent pas à une certaine unité, à des possibilités d'échanges interdisciplinaires et de réflexion sur les questions fondamentales, elle se condamne à dépérir, et le milieu québécois sera mal en point. L'avenir de la philosophie au Québec, c'est l'avenir du Québec. C'est aussi simple que ça quant à moi. 
En d'autres termes, la philosophie telle que je la vois, telle que je la considère, en toute humilité, a un rôle vraiment fondamental, un objectif extrêmement important. Si cela ne peut pas fonctionner, si effectivement son rôle est un rôle diminué qui ne s'exprime ou ne s'accomplit que partiellement, alors l'université et la société elle-même vont en souffrir. Par conséquent, la question qui se pose n'est pas seulement celle de l'avenir de la philosophie au Québec, mais plus fondamentalement celle de l'avenir du Québec et même au plan international, celle de l'avenir du monde. Cela peut paraître prétentieux mais cela ne l'est pas. Bien sûr, nous sommes des individus, mais ce que nous devons viser, c'est autre chose qu'une dispersion des efforts dans la création de petites chapelles d'influence, dans la constitution d'ensembles ou de complexes plus ou moins sophistiques comme à l'époque pré-socratique. Platon, parlant quelque part dans ses dialogues du sophiste Prodicos, nous dit qu'on n'entendait que le bourdonnement de sa voix sans pouvoir saisir ce qu'il disait. C'est ça effectivement la sophistique. C'est un discours qui ne vise pas la vérité. C'est un discours qui subordonne la recherche de la vérité, à autre chose, qui l'utilise, qui l'occulte en faveur de fins en elles-mêmes littéralement insignifiantes, telles que le prestige, le pouvoir, l'argent etc. C'est une déviation, un danger permanent pour la philosophie, que notre maître à tous, Socrate, a dénoncé avec vigueur...

En suivant à l'occasion quelques cours dans d'autres disciplines, j'ai réalisé que chaque discipline, lorsqu'elle arrive à un certain niveau de profondeur, débouche sur un questionnement philosophique qui lui donne un sens vraiment fondamental, que ce soit en sociologie, en service social, en psychiatrie. C'est une fonction à laquelle les philosophes ne peuvent se soustraire, une fonction essentielle à l'intérieur de l'université dans la mesure où elle est véritablement université. Il en est de même, sinon peut-être encore davantage, dans un collège, où la philosophie s'adresse à tout le monde, c'est-à-dire à l'ensemble des étudiants qui suivent des cours obligatoires de philosophie. Le rôle du professeur de philosophie de collège est peut-être encore beaucoup plus stable, beaucoup plus unificateur, dans le bon sens du terme, que celui du professeur d'université dont les étudiants sont cloisonnés dans un département, et qui s'adresse à un nombre relativement restreint d'étudiants. 
Les philosophes doivent répondre aux attentes du milieu dans la mesure de leurs possibilités, de leurs capacités. Ils doivent intervenir en profitant des occasions qui s'offrent à eux comme les congrès, les colloques qui sont des moyens de s'exprimer sur les problèmes qui se posent à divers niveaux.

\section{É.C.: Est-ce le mot de la fin?}

V.C.: Je dis parfois aux étudiants au début d'un cours qu'être philosophe ou tenter de le devenir, c'est extrêmement ambitieux, car on cherche à s'interroger sur ce qui est vraiment fondamental. Il faut avoir une bonne dose d'humilité, beaucoup de sincérité et de rectitude, et éviter tout ce qui porte atteinte à l'intégrité personnelle, comme de penser, comme on l'a fait parfois, que des moyens malhonnêtes peuvent trouver une justification dans la poursuite de fins prétendûment bonnes. Il y a tellement à faire dans l'enseignement collégial et universitaire, de même que pour contribuer à la solution des graves problèmes qui se posent au monde contemporain, qu'on n'aura pas trop des efforts de réflexion et des labeurs de tous ceux que passionne la philosophie...

É.C. : Monsieur Venant Cauchy, je vous remercie.

Entretien réalisé en décembre 1989. 